\title{
Aqua MODIS Electronic Crosstalk Survey: Mid-Wave Infrared Bands
}

\author{
Graziela R. Keller, Truman Wilson, Xu Geng, Aisheng Wu, Zhipeng Wang, and Xiaoxiong Xiong
}

\begin{abstract}
The Moderate Resolution Imaging Spectroradiometer (MODIS) on board NASA's Aqua polar-orbiting satellite has long been known to be subjected to electronic crosstalk. Its signatures are clearly visible in images of the lunar disk by various bands, which are routinely obtained during scheduled roll maneuvers. Electronic crosstalk can potentially impact Level $1 B$ products, causing image artifacts such as striping and radiometric bias. However, a comprehensive effort in mapping the sending/receiving bands and detectors, deriving the crosstalk coefficients, and assessing the impact of the crosstalk contamination on the Level 1B product for Aqua's mid-wave IR bands $(3.75-4.52 \mu \mathrm{m})$ had been lacking. In this work, we surveyed lunar images by the Aqua MODIS bands that are connected to electronic output 2 of the SWIR/MWIR FPA, identifying all detectors affected by electronic crosstalk contamination and determined which bands and detectors are sending the contaminating signal. By assuming a linear model to describe the contamination, we derived linear crosstalk coefficients for the bands/detectors concerned from lunar images and used these to generate corrected Earth Level 1B images for the mid-wave IR bands and assessed the impact of the electronic crosstalk on the Level 1B imagery.
\end{abstract}

Index Terms-Aqua, artifact, crosstalk, MODIS, Moon

\section{INTRODUCTION}

$\mathbf{T}$ HE MODIS instrument on board the low Earth orbit Aqua satellite, lauched in 2002, is a passive cross-track scanner (Fig.1) that images the Earth in 36 spectral bands. 16 of these are thermal emissive bands (TEBs), with center wavelengths ranging from 3.75 to $14.24 \mu \mathrm{m}$ and 20 are reflective solar bands (RSBs), with center wavelengths between 0.41 and $2.13 \mu \mathrm{m}$. Each of MODIS's individual bands is comprised by its own array of detectors, with each array being placed in one of four separate focal plane assemblies (FPAs): the visible (VIS), the near-infrared (NIR), the short- and mid-wave infrared (SWIR/MWIR), and the long-wave infrared (LWIR) FPAs. The arrays of detectors corresponding to each band are arranged side-by-side in the along-scan direction and observe the same region of the Earth consecutively as the scan mirror rotates.

As illustrated by the scheme in Fig. 2, during a 360 degree rotation of the double-sided scan mirror, which

Manuscript received XXXX XX, 2017; revised XXXX XX, 2017.

G. R. Keller, T. Wilson, X. Geng, and A. Wu are with Science Systems and Applications, Inc., Lanham, MD, 20706 USA (e-mail: graziela.keller@ssaihq.com, truman.wilson@ssaihq.com, xu.geng@ssaihq.com, and aisheng.wu@ssaihq.com)

$\mathrm{X}$. Xiong is with Sciences and Exploration Directorate, NASA Goddard Space Flight Center, Greenbelt, MD 20771 USA (e-mail: xiaoxiong$1 @$ nasa.gov)

Z. Wang was with Science Systems and Applications, Inc. while this work was developed and now is with Earth Resources Technology, Inc., (email: zhipeng.wang@noaa.gov)
TABLE I: Wavelength interval (WI), FPA, band type (RSB or TEB), and primary use for the bands connected to electronic output 2 of the SWIR/MWIR FPA.

\begin{tabular}{|c||c|c|c|c|}
\hline Band & WI $(\mu \mathrm{m})$ & Type & FPA & Primary Use \\
\hline \hline 20 & $3.660-3.840$ & & & \multirow{2}{*}{} \\
\cline { 1 - 2 } 21 & $3.929-3.989$ & \multirow{2}{*}{ TEB } & MWIR & Surface/Cloud Temperature \\
\cline { 1 - 2 } 22 & $3.929-3.989$ & & & \\
\hline 23 & $4.020-4.080$ & & & \\
\cline { 1 - 2 } 24 & $4.433-4.498$ & \multirow{2}{*}{ TEB } & MWIR & Atmospheric Temperature \\
\hline 25 & $4.482-4.549$ & & & \\
\hline 26 & $1.360-1.390$ & RSB & SWIR & Cirrus Clouds/Water Vapor \\
\hline
\end{tabular}

takes 2.954 seconds, the optical system views a series of onboard calibrators, as well as Earth scenes, consecutively. Within one swath, the Earth image, acquired within $\pm \mathbf{5 5}$ degrees from the Nadir direction, will contain 1354 frames per scan line. Consecutive scan lines are acquired through alternate sides of the scan mirror.

Fig. 3 illustrates the distribution of all 36 of MODIS bands within the four different FPAs. The separation between bands in each FPA is shown in terms of $1 \mathrm{~km}$ instantaneous field of view (IFOV) offsets along the scan direction. The science packets will be assembled with the bands being aligned by the these offsets, in order for them to view a common ground sample.

In each of the FPAs, the detectors are mated to a readout

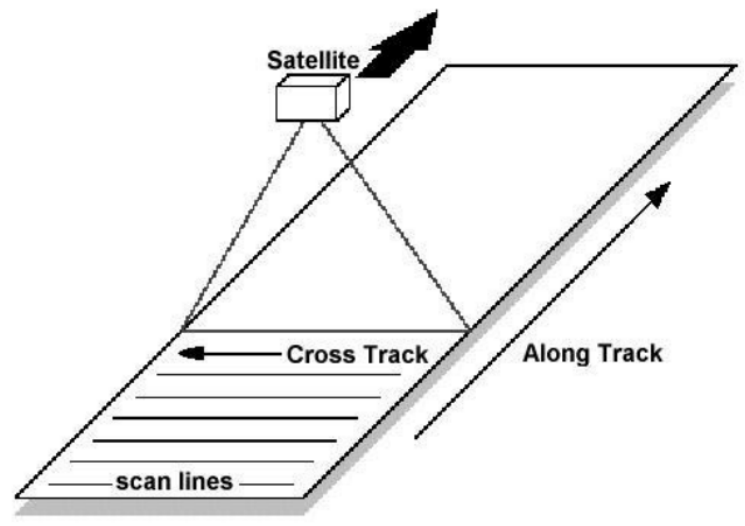

Fig. 1: Geometry of a MODIS observation swath, where the scan lines (in the along scan or cross-track direction) are approximately perpendicular to the direction of the motion of the satellite (along track direction). Figure taken from MODIS Level 1B Product User's Guide. 


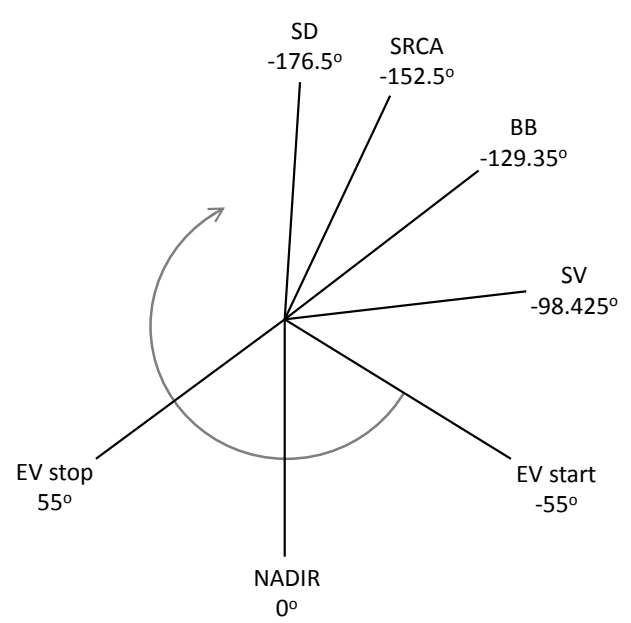

Fig. 2: Angle by which the mirror rotates, with respect to NADIR, when observing each of the on-board calibration targets - Solar Diffuser (SD), spectroradiometric calibration assembly (SRCA), black body (BB), space view port (SV) - and the Earth view (EV).

integrated circuit. The bands are further grouped together in electronic outputs, which dictate the order and timing of the signal readout. The bands within the same electronic output will be sampled consecutively and one detector at a time.

The space-view analog module (SAM) buffers and digitizes the analog signal produced by the photovoltaic (PV) FPAs (bands 1 to 30), converting it to 12 bit digital signals, which are then formatted into science data packets, buffered and sent to the spacecraft.

The MODIS's mid-wave IR bands we address in this paper -20 to $25-$, together with the short-wave IR band 26 (Table I), are all connected to electronic output 2 of the SWIR/MWIR FPA. These bands are comprised of single column arrays of 10 detectors with a $1 \mathrm{~km}$ footprint at nadir each and which will be identified here by numbers between 1 and 10 , in the so called "product order" (see, for example, [1] for details on the MODIS design).

Aqua MODIS, together with its predecessor on-board the Terra satellite, is a heritage Earth-observing sensor and one of the most important instruments monitoring Earth's oceans, land, and atmosphere. As such, it requires accurate and stable calibration. It carries a suite of on-board calibrators used to derive on-orbit calibration coefficients and to assess sensor stability [2], [3]. These measurements are further complemented by routine observations of the Moon during scheduled roll maneuvers [4]-[6], which have been historically used to support the on-orbit calibration and sensor characterization. However, signatures of electronic crosstalk contamination are clearly seen in images of the Moon from both Aqua and Terra MODIS.

In general terms, electronic crosstalk takes place when signal being transmitted in one circuit or part of a circuit creates an unsought effect in another circuit or part of a circuit. In the MODIS instruments, signal registered by some detectors of some bands (here termed the sending bands/detectors) will

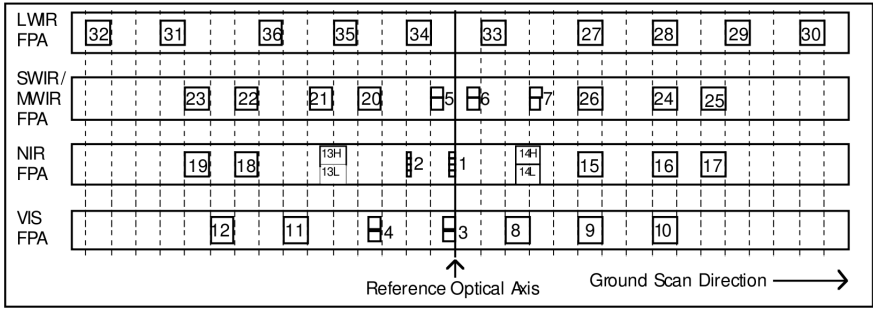

Fig. 3: The scheme illustrates how MODIS's 36 spectral bands are distributed withing the four separate FPAs. In each FPA, the arrays of detectors corresponding to each band are arranged side-by-side in the along-scan direction and observe the same Earth target consecutively, as the mirror rotates. The separation between bands in each FPA is shown in terms of $1 \mathrm{~km}$ IFOV offsets along the scan direction.

induce responses in other detectors (the receiving detectors), which may either belong to the same band or to different bands. In the cases addressed here, the sending and receiving bands are placed not only in the same focal plane assembly, but are also connected to the same electronic output.

Electronic crosstalk can potentially impact the Level 1B and higher level products, causing image artifacts such as striping and radiometric bias, as has been extensively described in the literature for Terra MODIS [7]-[12]. In Terra MODIS, the severity of the artifacts impacting the Level 1B imagery from the thermal emissive bands 27 to 30 - on the LWIR FPA prompted the MODIS Characterization Support Team (MCST) to implement a correction in Collection 6.1 [12].

Although the MODIS instruments have long been known to be subjected to electronic crosstalk and pre-launch tests have empirically outlined the nature of the crosstalk contamination, a comprehensive effort in mapping the sending/receiving bands and detectors, characterizing the crosstalk contamination, and assessing the impact on the Level 1B product for Aqua MODIS mid-wave bands had, so far, been lacking [13]-[15]. In [13], we linked bright stripes apparent in Level 1B images by Aqua MODIS band 24 to electronic crosstalk contamination in detector 1 and derived a successful correction. In the present paper, we expand on that investigation by surveying lunar images by all the Aqua MODIS bands that are connected to electronic output 2 of the SWIR/MWIR FPA, identifying all detectors affected by electronic crosstalk and mapping the contamination back to the respective sending bands and detectors. By assuming that the electronic crosstalk contamination is proportional to the signal of each sending band/detector combination, we derive linear crosstalk coefficients for the bands/detectors concerned, for the entire mission, and use these to generate corrected Level 1B images for the mid-wave IR bands (20 to 25) and assess the impact of the electronic crosstalk on the Level 1B imagery. While we do not address here the impact of the electronic crosstalk on the L1B product by short wave IR band 26 (which is a RSB, while bands 20 to 25 are TEBs), we do take this band into consideration in this analysis and calculate its crosstalk coefficients. We do this because, as we will describe ahead, this band is connected to the same electronic output as the mid-wave IR bands and is necessary for the understanding of 
the process underlying the pattern of contamination we see in the MODIS instruments. Terra and Aqua MODIS SWIR bands $5-7$ and 26 do have crosstalk correction implemented into the L1B product from the beginning of each mission [4].

The remainder of this paper is organized as follows. In Section II, we present the evidence of electronic crosstalk contamination in the Aqua MODIS bands connected to electronic output 2 of the SWIR/MWIR FPA, the so called crosstalk ghosts, which are seen in lunar images. In Section III, we introduce the model adopted to describe the contamination, identify the bands and detectors responsible for contaminating each of the receiving bands, and detail the algorithm used in the derivation of the crosstalk coefficients. In Section IV, we show examples of Level 1B granules that were corrected of crosstalk contamination and discuss its impact. In section $\mathbf{V}$, we discuss the main assumptions made in this work. We conclude in Section VI by identifying which of the receiving bands and detectors are most affected by the electronic crosstalk contamination and constitute good candidates for further testing and a possible implementation of the electronic crosstalk correction into a future Collection.

\section{EVIDENCE FROM LUNAR IMAGES}

Aqua MODIS acquires, on average, around 9 scheduled lunar observations a year, which are routinely used to support RSB radiometric calibration. During these events, a roll maneuver is performed and the Moon is viewed through the space-view port. The data, however, is stored in the earth-view data sector, where the Moon appears in several consecutive scans, co-registered in all bands. The scheme in Fig. 4 shows the layout of SWIR/MWIR FPA with a representation of the image of the Moon superimposed and to scale.

Differently from Nadir Earth observations, which are seamless between consecutive scans, when the MODIS instruments observe the Moon, the images of the lunar disk from consecutive scans overlap to some extent [16]. This oversampling of the lunar disk, combined with the fact that observations made by several consecutive scans, at slightly different positions on the detectors, are available, makes it possible to create images of the entire Moon by selecting only pixels from a single detector of a given band. Because the Moon is an isolated, high-contrast target of known shape, lunar observations produce the best images for identifying signs of electronic crosstalk contamination and the single detector images allow for the contamination in each of the detectors to be analyzed separately.

The crosstalk artifacts, which we refer to as crosstalk ghosts, consist of attenuated images of the target as seen by the band/detector or bands/detectors sending the contaminating signal, superimposed on the image of the detector that receives the contamination. The electronic crosstalk ghosts appear dislocated with respect to the main image in both the track and the scan directions, with the displacement in the scan direction corresponding to the distance between sending and receiving bands in the FPA and the displacement in the track direction depending on which detectors are sending and receiving the contamination.
SWIR/MWIR FPA

\begin{tabular}{|c|c|c|c|c|c|c|c|c|c|c|}
\hline \multirow{2}{*}{ Scan } & B25 & B24 & B26 & \multirow{11}{*}{$\begin{array}{l}\text { B7 } \\
1 \\
- \\
- \\
- \\
- \\
- \\
- \\
- \\
20\end{array}$} & \multirow{11}{*}{$\begin{array}{l}\text { B6 } \\
1 \\
- \\
- \\
- \\
- \\
- \\
- \\
-\end{array}$} & \multirow{11}{*}{$\begin{array}{l}\text { B5 } \\
1 \\
-1 \\
- \\
- \\
- \\
- \\
\\
\\
20\end{array}$} & $B 20$ & B2 & R2 & $B 23$ \\
\hline & 1 & 1 & 1 & & & & 1 & 1 & 1 & 1 \\
\hline \multirow{9}{*}{ ฟ } & 2 & 2 & 2 & & & & 2 & 2 & 2 & 2 \\
\hline & 3 & 3 & 3 & & & & 3 & 3 & 3 & 3 \\
\hline & 4 & 4 & 4 & & & & 4 & 4 & 4 & 4 \\
\hline & 5 & 5 & 5 & & & & 5 & 5 & 5 & 5 \\
\hline & 6 & 6 & 6 & & & & 6 & 6 & 6 & 6 \\
\hline & 7 & 7 & 7 & & & & 7 & 7 & 7 & 7 \\
\hline & 8 & 8 & 8 & & & & 8 & 8 & 8 & 8 \\
\hline & 9 & 9 & 9 & & & & 9 & 9 & 9 & 9 \\
\hline & 10 & 10 & 10 & & & & 10 & 10 & 10 & 10 \\
\hline
\end{tabular}

Fig. 4: Scheme showing the layout of the SWIR/MWIR FPA with a representation of the image of the lunar disk superimposed. The detectors, indicated by numbers 1 to 20 for bands $5,6,7$ and 1 to 10 for all other bands, are shown in 'product order'. While bands 20 to 26 are connected to electronic output 2 of the SWIR/MWIR FPA, bands 5 to 7 are connected to output 1 of the same FPA. Scheme based on figure 1 from [17].

In this work, we address two distinct manifestations of electronic crosstalk unveiled while inspecting Aqua MODIS lunar images from the scheduled lunar events, to which we will refer here as the simple and the lateral ghosts. The simple ghosts consist of a positive contamination caused by only one sending detector of one sending band and which, for this reason, are shaped similarly to the main Moon image. The
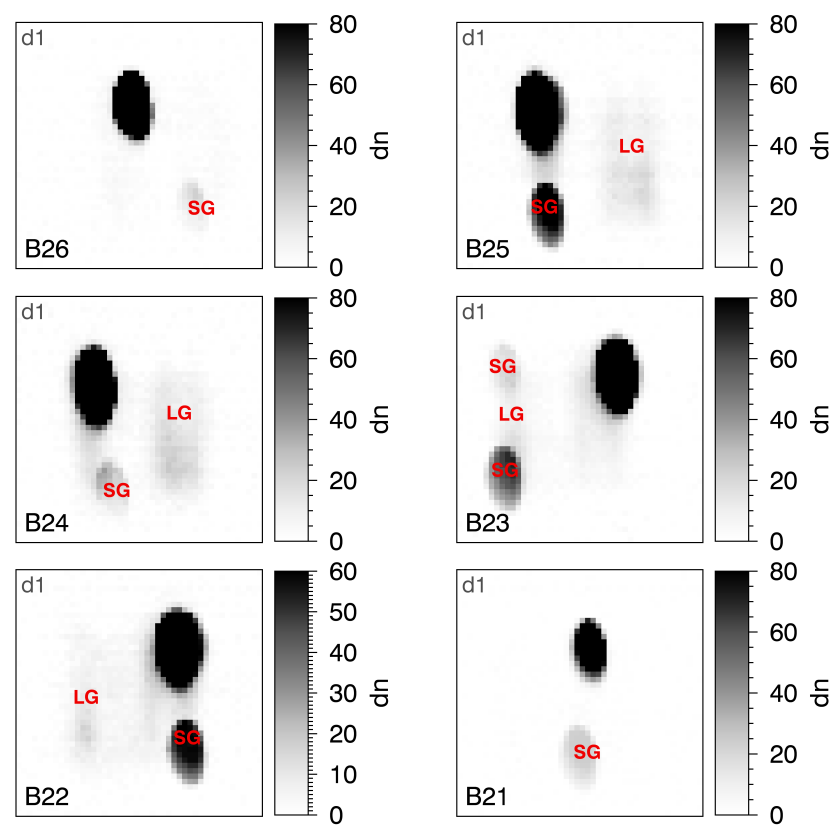

Fig. 5: Lunar images from detector 1 of bands 26 to 21 (from top left to bottom right) showing the main lunar image and crosstalk ghosts (band 20 was not shown because, as explained in the text, the lateral ghosts are very faint and difficult to show as images). Simple ghosts (SG) only affect the detector 1 of the receiving bands 20 to 26 . The lateral ghosts (LG) are present in all the detectors of bands $20,22,23,24$, and 25 . dn, in the colorbar title, refers to the background subtracted instrument response and the scale was truncated to facilitate the visualization of the crosstalk ghosts which are much fainter than the main lunar profile. 
lateral ghosts we address in this work, on the other hand, are seen as extended regions of faint, positive signal next to the main lunar images, which we find to be caused by multiple sending bands and detectors. Fig. 5 shows examples of both simple and lateral ghosts seen in single detector lunar images. The simple ghosts only affect the detector 1 of the receiving bands 20 to 26 . The fainter lateral ghosts, on the other hand, are visible in images from all the detectors of bands 20, 22, 23,24 , and 25 .

\section{ChARACTERIZATION}

\section{A. Model}

In this work, we follow the literature and describe the crosstalk contamination according to (1), where we assume each sending detector produces a contamination proportional to its signal [7]-[12].

$$
d n_{i}(S, F)=d n_{i}^{*}(S, F)-\sum_{j} c_{i, j} \times d n_{j}\left(S, F+\Delta F_{j}\right)
$$

Here, $d n$ is the background subtracted instrument response of the receiving and sending bands and the symbol * indicates the contaminated signal of the receiving band. The subscripts $i$ and $j$ are the indexes for the receiving and sending detectors, respectively. $c_{i, j}$ is the so called crosstalk coefficient for each pair of receiving/sending detectors. Here, we postulate $c_{i, j}=0$ when $i=j$, as having a detector inducing a crosstalk contamination in itself is not something we can measure as the ghost and main lunar images would align. In any case, regular calibration is expected to be able to absorb this effect - if it were present - which would simply mimic a detector with a somewhat different response. The capital letters $S$ and $F$ denote scan and frame numbers, respectively, and $\Delta F$ denotes the displacement in the frame direction, which corresponds to the separation between the sending and receiving bands on the focal plane assembly.

It is relatively easy to know, in lunar images, what the non-contaminated background subtracted instrument response should be in the position of the ghost were crosstalk not present (that is, the left term in (1)). In the case of the crosstalk ghosts addressed in this paper, because they appear sufficiently displaced from the main lunar image, it is reasonable to assume $d n_{i}(S, F)=0$ and solve the equation for every pixel of the ghost image, deriving the crosstalk coefficients that will then be applied in the correction of the L1A Earth and calibration images, to produce corrected Level 1B images.

TABLE II: Electronic crosstalk sending/receiving band/detector combinations for the detector 1 simple ghosts.

\begin{tabular}{|c||c|c|c|c|c|c|c|c|}
\hline \multicolumn{1}{|c||}{ FPA } & \multicolumn{7}{c|}{ SWIR/MWIR } \\
\hline Receiving Band & 20 & 21 & 22 & 23 & 23 & 24 & 25 & 26 \\
Receiving Detector & 1 & 1 & 1 & 1 & 1 & 1 & 1 & 1 \\
\hline Sending Band & 22 & 20 & 23 & 25 & 25 & 26 & 24 & 21 \\
Sending Detector & 10 & 10 & 10 & 10 & 1 & 10 & 10 & 10 \\
\hline
\end{tabular}

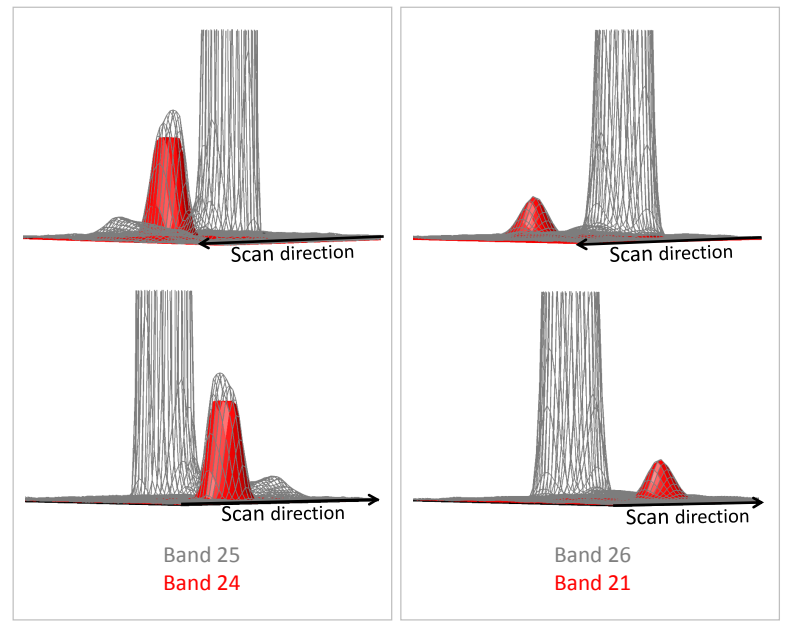

Fig. 6: Examples of simple ghosts seen in the detector 1 images of the receiving bands 25 and 26 (gray) are shown as surface plots in two differenf perspectives. In red, the scaled images of the detector 10 of the sending bands ( 24 and 21, respectively - red) align with the ghost image after the appropriate displacements in the scan direction are applied, which corresponds to the distances between the sending and receiving bands in the FPA. Notice that the band 24's profile in the image on the left is saturated, while the profile of the ghost is not (see text). The z-axes of the surface plots were truncated (at 150 counts for band 25 and 50 counts for band 26) to enable the visualization of the faint ghosts. In the image by band 25, the lateral ghost is also seen.

\section{B. Simple Ghosts}

The simple ghosts are seen in detector 1 images from bands 20 to 26 (Fig. 6) and are absent from images by other detectors. We determined the bands and detectors sending the contamination to the detector 1 of each of the receiving bands, by visual inspection. The lunar profile in the single detector image of the sending band/detector will align (after being displaced by the correct amount in the scan direction corresponding to the distance between bands in the FPA) with that of the ghost in the single detector image of the receiving band. Table II lists the pairs of sending/receiving bands/detectors we identified for the detector 1 simple ghosts affecting bands on the output 2 of the SWIR/MWIR FPA.

We find that while the detector 1 simple ghost of each receiving band is caused by a different sending band, the sending detectors are always the detector 10 of the respective sending band, with the only exception being an extra ghost on detector 1 images of band 23, which is caused by signal coming from the detector 1 of the same sending band (25). This extra ghost is, however, much fainter than the one caused

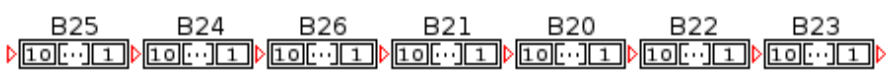

Fig. 7: Read out order for bands in output 2 of the SWIR/MWIR FPA, starting from left to right. In the SWIR/MWIR FPA, Band 25 is read again, after Band 23. Bands 5, 6, and 7, while also in the SWIR/MWIR FPA, are not connected to the same electronic output. 


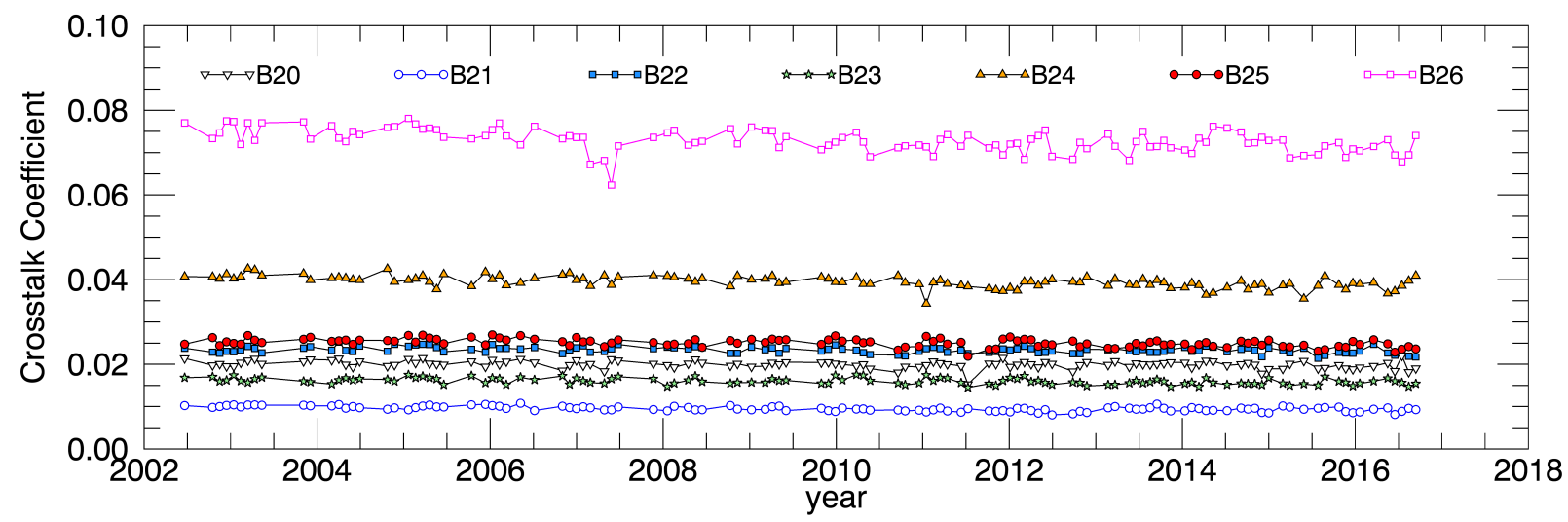

Fig. 8: Simple ghost crosstalk coefficients for bands in the SWIR/MWIR FPA were derived for scheduled lunar events over the entire mission, for the detector 1 of the receiving bands (corresponding sending bands in Table II).

by signal from sending detector 10 . None of the other bands connected to electronic output 2 of the SWIR/MWIR FPA show this additional ghost.

The scheme in Fig. 7 illustrates the order of signal readout for the bands connected to electronic output 2 of the SWIR/MWIR FPA. If we consider the band/detector order with which the signal of each of the pairs of sending/receiving detectors is read out, we find that detector 1 of the receiving band is always read immediately before detector 10 of the sending band. This scenario points towards a common underlying process responsible for this pattern of crosstalk contamination in which we have the signal from detector 10 of the sending band contaminating detector 1 of the receiving band. One interpretation would be a failure in stopping the receiving band from being read out by the time the signal from the next detector - from the sending band - starts being sampled.

The crosstalk coefficients for the simple ghosts are derived from (1). While this equation allows for each detector to be contaminated by multiple detectors from multiple sending bands, when deriving the crosstalk coefficients for the simple ghosts, where each receiving detectors from each receiving band is contaminated by signal coming from only one detector of only one sending band, the sum in the second term on the right-hand side of (1) is dropped [13]-[15]. We assume the term on the left side of the equation is zero at the position of the ghosts, in the lunar images. We then solve the equation for every pixel of the ghost image, and take the crosstalk coefficient to be the average value. In some instances, other artifacts will be present in the image that may overlap with the ghost, in which case care is necessary to avoid the affected pixels. Another difficulty is that all of Aqua's thermal emissive bands (excluding the fire band 21) saturate when imaging the Moon during the scheduled lunar events. Interestingly, the electronic crosstalk ghost retains the correct shape (as can be seen in Fig. 6), which implies that the electronic crosstalk contamination takes place before the analog-digital conversion stage where the saturation occurs. Thus, in the case of the simple ghosts, saturated pixels were not used in all cases where the sending band signal saturates. For the receiving bands 24 and 26 this was not a problem because their sending bands 26 and 21 , respectively - do not.

Fig. 8 shows the derived crosstalk coefficients for the entire mission, for the detector 1 simple ghosts that were caused by contamination originating from detector 10 of the sending band. Each point of the curves corresponds to one scheduled lunar event. The plot shows that the electronic crosstalk mechanism responsible for the simple detector 1 ghosts has been present since the beginning of the mission, in 2002, and that the crosstalk coefficients have all been very stable, with only slight downward trends.

\section{Lateral Ghosts}

Lateral ghosts consisting of faint extended regions of positive signal next to the main lunar image are seen in single detector lunar images by bands 20, 22, 23, 24, and 25. Unlike the simple detector 1 ghosts, these are present in images by all of the detectors of the affected bands. They are caused by signal from multiple detectors of multiple sending bands.

Each sending detector will contaminate some region of the receiving image and because there are multiple detectors sending signal and because the ghost images may partially overlap, the lateral ghosts will have complicated shapes. It is known that the position of the contamination depends both on the sending band and on the sending detector. Thus, the translation on the scan direction of the single detector image of the Moon by a given sending band/detector will align it with its ghost in the receiving image.

The crosstalk coefficients for the lateral ghosts are derived from (1), again, assuming the term on the left side of the equation to be zero. Here, however, we solve the equation for each of the receiving detectors using multiple linear regression, since the problem is equivalent to having

$$
y_{i}=c_{1} x_{i 1}+c_{2} x_{i 2}+c_{3} x_{i 3}+\ldots+c_{p} x_{i p},
$$

for $i=1,2,3, \ldots, n$ observations, where $x$ and $y$ refer to the independent and dependent variables, respectively, for a number $p$ of explanatory variables, corresponding to the number of sending detectors. The number of observations $n$ 
TABLE III: Electronic crosstalk sending/receiving bands for the lateral ghosts.

\begin{tabular}{|c||c|c|c|c|c|}
\hline \multicolumn{1}{|c||}{ FPA } & \multicolumn{5}{c|}{ MWIR } \\
\hline Receiving Band & 20 & 22 & 23 & 24 & 25 \\
\hline Sending Bands & 24,25 & 24,25 & 24,25 & $20-23$ & $20-23$ \\
\hline
\end{tabular}

corresponds to the number of image pixels comprised by the ghost.

The maximum number of possible sending detectors is 70, corresponding to 10 detectors from 7 bands. However, some of these sending bands correspond to positions in the receiving image, where no crosstalk ghosts is actually present, in which case the algorithm would deem them as not sending, and their crosstalk coefficients would return zero. A typical number of pixels contained within a lateral ghost for band 24, for example, is $\mathbf{4 5 0}$ pixels which constrain 40 parameters, given that receiving band 24 is found to be contaminated (in the case of the lateral ghost) by only 4 bands (with 10 detectors each).

Table III lists the sending bands responsible for the lateral ghosts present in each of the receiving bands. While the receiving bands 24 and 25 are contaminated by signal coming from bands 20,21, 22, and 23, the receiving bands 20, 22, and 23 are contaminated by signal from bands 24 and 25 . Fig. 9 illustrates this scenario. It shows single detector images of the receiving bands as gray surfaces. The contamination by each of the sending bands is also shown, according to the color code on the plot on the left hand side. The plots on the right show the total contamination by all sending bands (given by the rightmost term of (1), once the crosstalk coefficients are known), which reproduce the profiles of the lateral ghost in the images of the receiving bands.

Because we have seen that the simple ghosts do not show saturated profiles, even when their sending bands do, we assume that the same will apply to the lateral ghosts. The shape of the lateral ghosts is given by a combination of ghosts from multiple sending detectors and the presence or absence of a saturated profile is not evident as it is for the simple ghosts. While in the case of the simple ghosts we merely removed saturated pixels from the calculation, the same approach would vastly complicate the algorithm to derive the crosstalk coefficients from the lateral ghosts. We thus reconstruct the saturated profiles of the sending bands assuming they have the same shape (though not intensity) as that of band 21 (the only Aqua MODIS TEB whose image of the Moon does not saturate).

Fig. 10 shows the derived crosstalk coefficients for the entire mission, for the lateral ghosts in the receiving bands $25,24,23$, 22 and 20. Each of the panels corresponds to one receiving detector and on the various curves of each plot, the points correspond to individual lunar events. We only show plots for the receiving detectors 1 to 3 of each receiving band, because the crosstalk coefficients for the remaining detectors behave very much like those for the first three detectors (with the exception of the simple ghosts in detector 1) in terms of both magnitude and stability. Even though we derived the lateral ghosts crosstalk coefficients individually for every

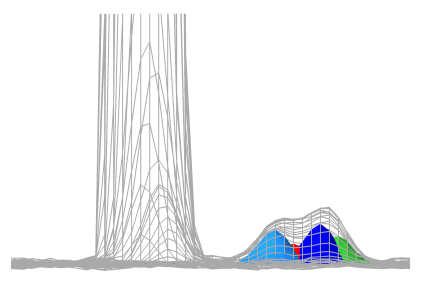

B25B20B21 B22 B23

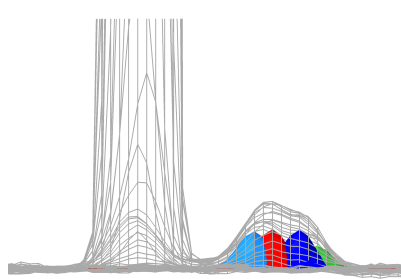

B24B20B21 B22B23

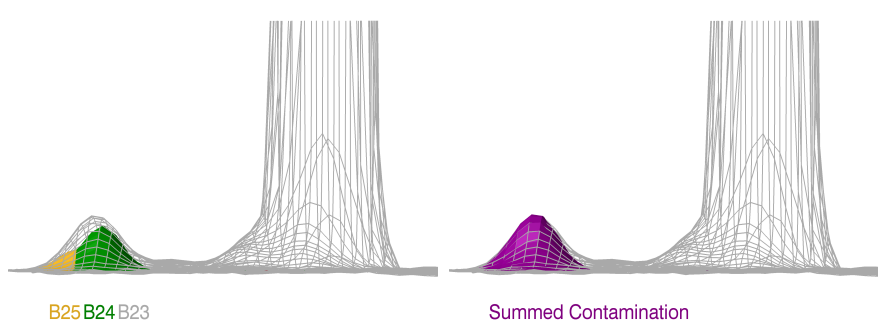

Fig. 9: Single detector images of the receiving bands 25, 24, and 23 (from top to bottom) are shown in gray, as surface plots, in the left and right panels. The vertical axes were truncated at $\mathrm{dn}=70$. Left: The contamination by each band on the region of the lateral ghost according to the color code. We find that the lateral ghosts present in single detector images from bands 25 and 24 are caused by contamination coming from bands 20,21, 22 and 23 (light blue, red, blue, and light green, respectively) and that the lateral ghosts present in single detector images from band 23 are caused by contamination coming from bands 24 and 25 (green and yellow, respectively). Right: in each panel, the combined contamination of the sending bands is shown as a purple surface and reproduces the profile of the lateral ghost in the image of the receiving band.

sending detector of every sending band contaminating each receiving detector of each receiving band, we show them here as averages of all the detectors in a sending band for simplicity. In the panels corresponding to the detector 1 , the crosstalk coefficients for the simple ghosts - derived separately and described above - are also shown in cyan. For band 23 , an additional simple ghost, caused by signal from the detector 1 of band 25, is present and its crosstalk coefficients are also shown, in purple. The crosstalk coefficients for this additional simple ghost were derived together with the lateral ones, due to the proximity between it and a lateral ghost and the fact that this simple ghost presents very faint signal, which would not allow it to be clearly separated otherwise.

In the case of the receiving bands 25 and 24, the crosstalk coefficients for sending band 21 are considerably larger than those of the other sending bands. However, this does not mean that band 21 is the main contributor for the signal of the lateral 
ghosts, as one can see in Fig. 9. The reason for this is the fact that band 21 is a low gain fire detection band, whose overall image counts are a lot lower than those registered in other bands, which means that even very large crosstalk coefficients may not result in a large contribution to the contamination. As an example, a sample granule over the African desert during summertime, a typically hot scene for band 21 , has a histogram which peaks around 60 counts (dn) and falls sharply towards higher values. A band 21 pixel registering 60 counts would cause a contamination in receiving band 25 , for example, of less than one count. Band 21 pixels over fire will register much higher values, of hundreds of counts, but they are few.

The crosstalk coefficients derived for the lateral ghosts in the various receiving bands are all very stable in time and indicate that the contamination was present since the beginning of the mission, similarly to what we have seen in the case of the simple ghosts. The exception to this is, perhaps, in the lateral ghost coefficients for receiving band 20, from sending band 24, which oscillate. However, the lateral ghosts in band 20 images are the weakest and are just about in the limit of what is possible for us to measure. The oscillatory behavior is thus likely to be a reflection of the difficulty in measuring such weak signals.

Fig. 11 shows sample lunar images before and after electronic crosstalk correction is applied and illustrates the capability of the model and regression algorithm in reproducing the contamination based in contributions from the sending bands. The average absolute response of the corrected image in the contamination region for all bands was below 0.05 counts, for the sample observation. The standard deviation of the same pixels for bands 25, 24, 23,22 , and 20 were $1.18,1.22,1.13,1.00,1.32$ counts, respectively.

\section{IMPACT ON LEVEL 1B EARTH IMAGES}

The simple and lateral ghosts seen in images of the Moon are expected to also affect Earth images and will do so in two different ways. Whenever only one or some of the detectors of a receiving band are affected by the electronic crosstalk contamination (as is the case of the simple ghosts that only affect the detector 1 of the receiving bands), or when some detectors are affected more than others, we can expect to see striping in Earth images. When, on the other hand, we have all the detectors of a band receiving somewhat similar amounts of contamination, as is the case with the lateral ghosts in this work, the Level 1B image is expected to present an overall radiometric bias.

In this work, we used the electronic crosstalk coefficients derived from the Moon images for both the lateral and the simple ghosts in the correction of sample Earth and calibration images, which allowed us to produce new, crosstalk corrected Level 1B images. MODIS TEBs are calibrated on-orbit using an on-board Black Body (BB), which is kept at $285 \mathrm{~K}$ during normal operations. For all TEBs, with the exception of band 21, BB images are used to derive the linear calibration coefficients (usually referred to as b1) at every scan. Scheduled BB warm-up/cool-down cycles take place every three months and are used to derive the on-orbit off-set $\left(\mathrm{a}_{0}\right)$ and non-linear $\left(a_{2}\right)$ calibration coefficients and the linear coefficients for band 21. In order to produce newly corrected Level 1B images, we also need to derive new crosstalk corrected b1 coefficients from BB observations. In this work we have not assessed the impact of the crosstalk contamination on the $a_{0}$ and $a_{2}$ coefficients, which have a lesser impact on the calibration.

Fig.s 12 to 15 illustrate the results we obtained for the receiving bands $25,24,23,22$, and 20, all of which show both lateral and simple electronic crosstalk ghosts in their lunar images. The crosstalk correction we applied to the Level 1B granules both mitigated striping artifacts associated with the simple ghosts and produced radiometric shifts, associated with the lateral ghosts. We found that, by far, the mid-wave IR band most affected by the crosstalk contamination was band 24 , both in terms of striping and of radiometric bias. We find the impact on bands 22 and 20 to be minor.

The severity of the electronic crosstalk artifacts in Level 1B imagery will depend not only on the absolute value of the signal leak and how it compares to the intensity levels in the receiving image, but also on the ability of the calibration to absorb the contamination. When the sending and receiving bands are close enough in wavelength that their images show similar patterns, the calibration will lessen the impact on the product more than when bands displaying contrasting images contaminate one another. The latter is the case of the contamination on detector 1 of receiving band 24 . Band 24 is a thermal emissive band, whose first detector is contaminated with signal coming from the detector 10 of band 26, a reflective solar band. This causes Aqua MODIS band 24 Level 1B images to display bright detector 1 stripes, mostly on darker regions of the image, a behavior that was previously characterized and corrected by us in [13]. In this work, we included the lateral ghosts into the analysis and the results now show not only the same striking reduction of the striping in the image, but also a considerable overall downward radiometric shift - of up to $1 \mathrm{~K}$ for the lowest brightness temperatures and $2 \mathrm{~K}$ at the highest end of the distribution, for the granules examined by us. An example of this behavior can be seen in the histograms and profile plots on Fig. 13

The striping associated with detector 1 is much less conspicuous in the other mid-wave IR TEBs than it is in band 24. In Figures 12 and 14, bands 25 and 23 show, respectively, striping artifacts with amplitudes of about $0.5 \mathrm{~K}$, which were successfully mitigated by the correction that was implemented. As can be seen in Fig. 15, the striping seen in the sample granules for bands 22 and 20 is quite insignificant. For band 21 , which only shows simple ghosts in its lunar images and no lateral ghosts, striping associated with the detector 1 can be seen in Level 1B imagery. However, band 21, which is a low gain fire detection band, has very noisy images, with detector histograms presenting considerable overall spread of all the detectors, which is typically more severe than the shift achieved by the crosstalk correction applied to detector 1 and was not, for this reason, shown here. In fact, Aqua MODIS band 21 not only has a noise requirement a lot less stringent than all the other TEBs $(2 \mathrm{~K})$, it also has three detectors currently flagged as noisy (detectors 2, 3, and 9) by the MCST. 

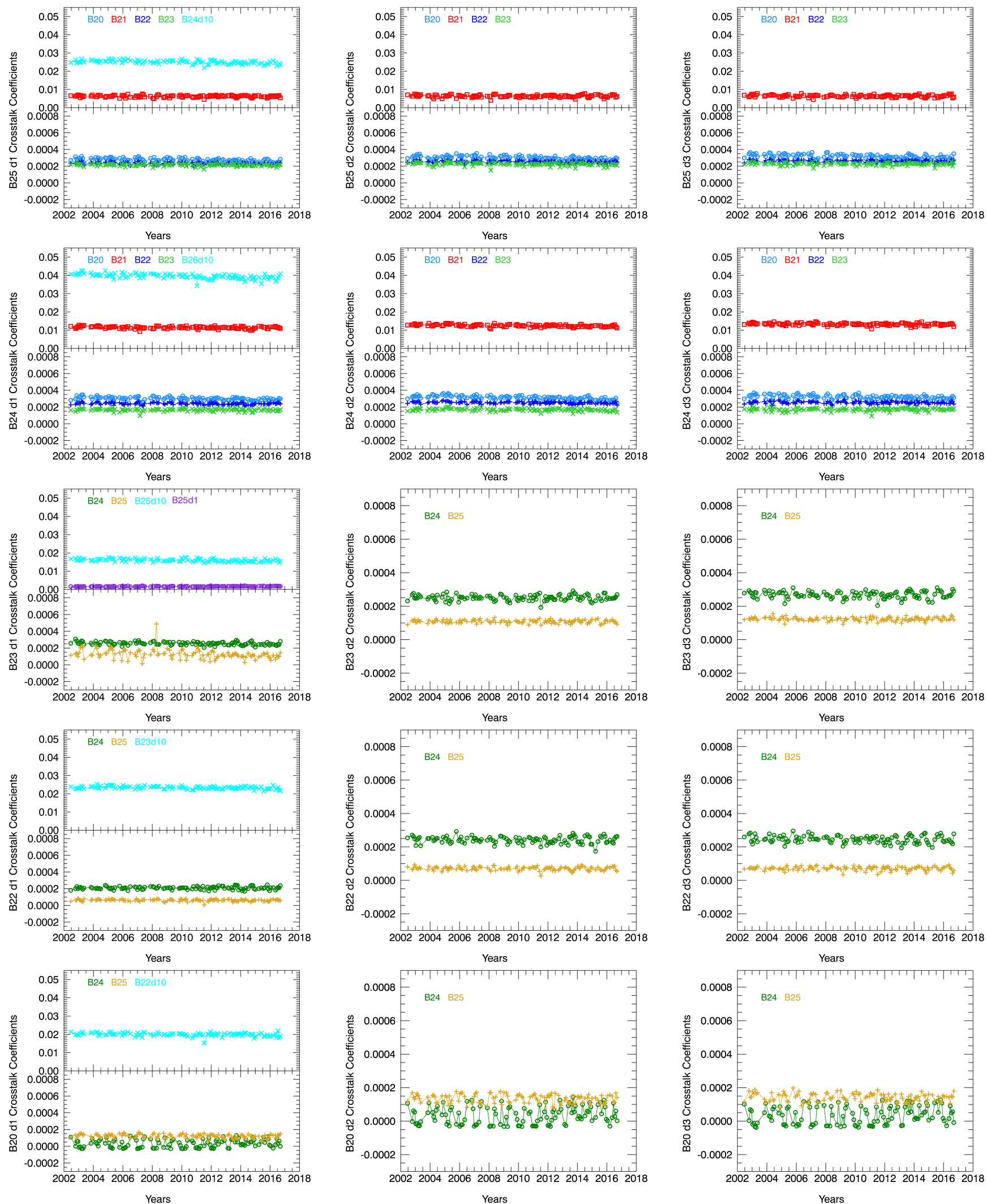

Fig. 10: Band averaged crosstalk coefficients for lateral ghosts of receiving bands 25, 24, 23, 22, and 20 (from top to bottom) derived from Moon images from scheduled lunar events. Each panel corresponds to one receiving detector. We only show plots for the receiving detectors 1 to 3 (from left to right) of each receiving band, since the lateral ghosts crosstalk coefficients for the remaining detectors behave similarly to those for the first three detectors in both magnitude and stability. For every receiving detector, we calculated crosstalk coefficients for each of the sending bands: B20 (light blue circles), B21 (red squares), B22 (blue +), and B23 (light green x), B24 (green circles), and B25 $($ yellow + ). For receiving detector 1 , the crosstalk coefficients for the simple ghost (cyan $\mathrm{x}$ ), which were derived separately, are also shown. The coefficients for the additional simple ghost in B23 are show as purple circles. 

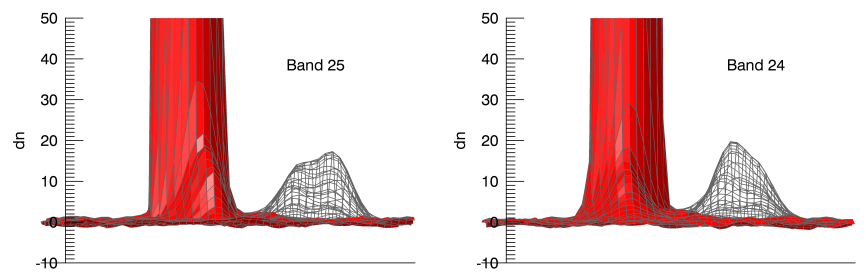

Fig. 11: Sample lunar images from bands 24 (left panel) and 25 (right panel) before (gray surface) and after (red surface) electronic crosstalk correction is applied. $d n$ refers to the background subtracted instrument response. The surfaces were truncated in the z-axes in order to allow the visualization of the faint ghosts. MODIS bands saturate at 4095 raw digital counts and in the case of band 24, for example, the background signal close to the main lunar images is around 325 counts.

The overall radiometric shifts shown by the mid-wave IR bands other than band 24 , are again much smaller than what was seen in this band. We found shifts of about $0.3 \mathrm{~K}$ for band 25 and $0.25 \mathrm{~K}$ for band 23. For bands 22 and 20, the shifts were even smaller. Unlike the other mid-wave IR bands, band 21 does not present contamination of all its detectors and thus does not present an overall electronic crosstalk related radiometric shift.

\section{DISCUSSION}

Two main assumptions made in this work are that the electronic crosstalk contamination depends on the signal of the sending detector linearly and that crosstalk coefficients obtained from Moon observations can be used to correct Earth images. Both assumptions are, of course, not necessarily true, but constitute natural initial assumptions that should only be further elaborated shall evidence of their inadequacy arise. The main argument in their favor is, in both cases, that these assumptions have allowed us and other authors [7], [11]-[15] to correct obvious artifacts in Earth images by both Aqua and Terra MODIS. In the case of the present work, detector 1 striping artifacts affecting various bands were satisfactorily corrected. These results indicate that the electronic crosstalk phenomena behave sufficiently similarly during both Earth and lunar observations and that the linear model is a sufficient approximation.

While it is easy to assess the success of the correction of striping artifacts in the imagery by evaluating how well the response of a detector agrees with that of its neighbors, the existence of an overall radiometric shift is more difficult to confirm. However, the fact that we found the temperature values retrieved from all detectors of a given receiving band to be shifted in such a fashion where not only the detector 1 striping was corrected, but also the introduction of new striping artifacts was avoided, is evidence in favor of the correction.

In our case, where the contamination - and thus the expected radiometric shift - is stable with time, long term brightness temperature trending over pseudo-invariant sites cannot offer clues. For Terra MODIS, however, the impact of the crosstalk on the photovoltaic LWIR bands (27 to 30) has been highly variable and long term trending offered a crucial test of the validity of the correction [12]. Another way of confirming the existence of the radiometric shift is through instrument intercomparison. However, we cannot expect to resolve small shifts of less than $1 \mathrm{~K}$ such as the ones we see for bands 20 , 22, 23, and 25. For band 24, however, where larger shifts are seen, instrument intercomparison can potentially be used to further validate our results and assumptions.

An instrument intercomparison between the MODIS TEBs and the Infrared Atmospheric Sounding Interferometer (IASI) was performed in [18], for MODIS Collections 5 and 6. The authors found that, for Aqua MODIS Collection 6 (Fig. 3 of their paper), band 24 presents an increased response with respect to that of IASI for higher brightness temperatures. In fact, band 24 was the only mid-wave IR band to show such a distinct trend, which qualitatively agrees with our results, where a greater downward shift is predicted for larger temperatures after correction. A quantitative validation would, however, require us to take into consideration the exact scene types being used in the comparison, as the level of crosstalk contamination depends on the scene. For example, band 24 does not present detector 1 stripes in night granules, as it is contaminated by signal coming from band 26 , an RSB that registers only low level noise during nighttime. Contamination originating in TEBs, on the other hand, will be stronger in hotter scenes, at lower latitudes. Since simultaneous intercomparison with IASI are only available for high latitude regions, results from scenes with high brightness temperatures are very limited. An intercomparison with the Atmospheric Infrared Sounder (AIRS), also on-board the Aqua satellite, would provide a wider temperature range and is likely to be pursued by the MCST in the near future.

Although the available evidence indicates that the assumptions made are sufficient, should further investigation point otherwise, models of greater complexity may be developed.

\section{CONCLUSiON}

In this paper, we surveyed lunar images from the Aqua MODIS bands that are connected to electronic output 2 of the SWIR/MWIR FPA, looking for signs of electronic crosstalk contamination. We identified the affected detectors and mapped the contamination back to the sending bands and detectors. By describing the contaminating signal using a linear model, we derived crosstalk coefficients from the lunar images and used these in the correction of L1B imagery of the mid-wave IR TEBs (bands 20 to 25), in order to assess the impact of the electronic crosstalk on the product.

Here, we described two distinct manifestations of electronic crosstalk responsible for causing distinct signatures in lunar images. We termed these the simple and lateral crosstalk ghosts. The simple ghosts affect only detector 1 of all the bands connected to electronic output 2 of the SWIR/MWIR FPA (i.e. bands 20 to 26). The contamination originates in only one detector of one sending band and even though each receiving band is contaminated by a different sending band (in the case of the simple ghosts only), the detector sending 
2002185.1735

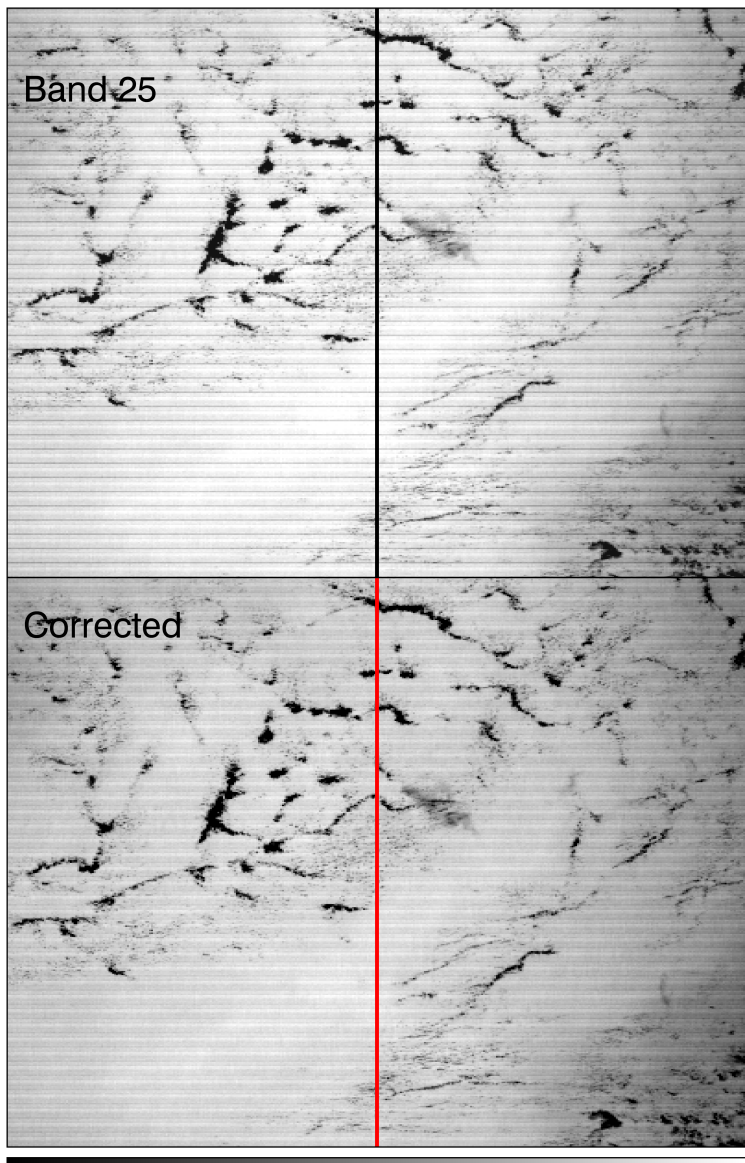

$271.0271 .5 \quad 272.0 \quad 272.5 \quad 273.0 \quad 273.5$ Brightness Temperature $(\mathrm{K})$
2002185.1735

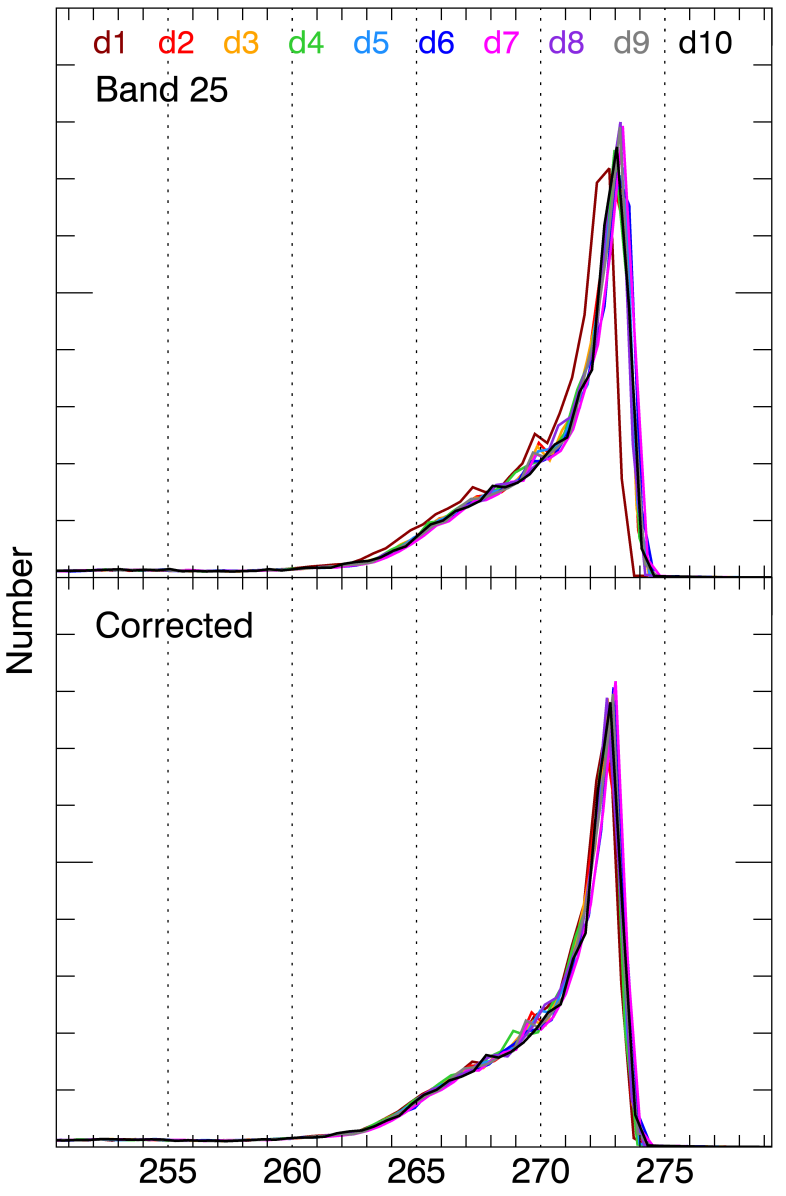

Brightness Temperature (K)

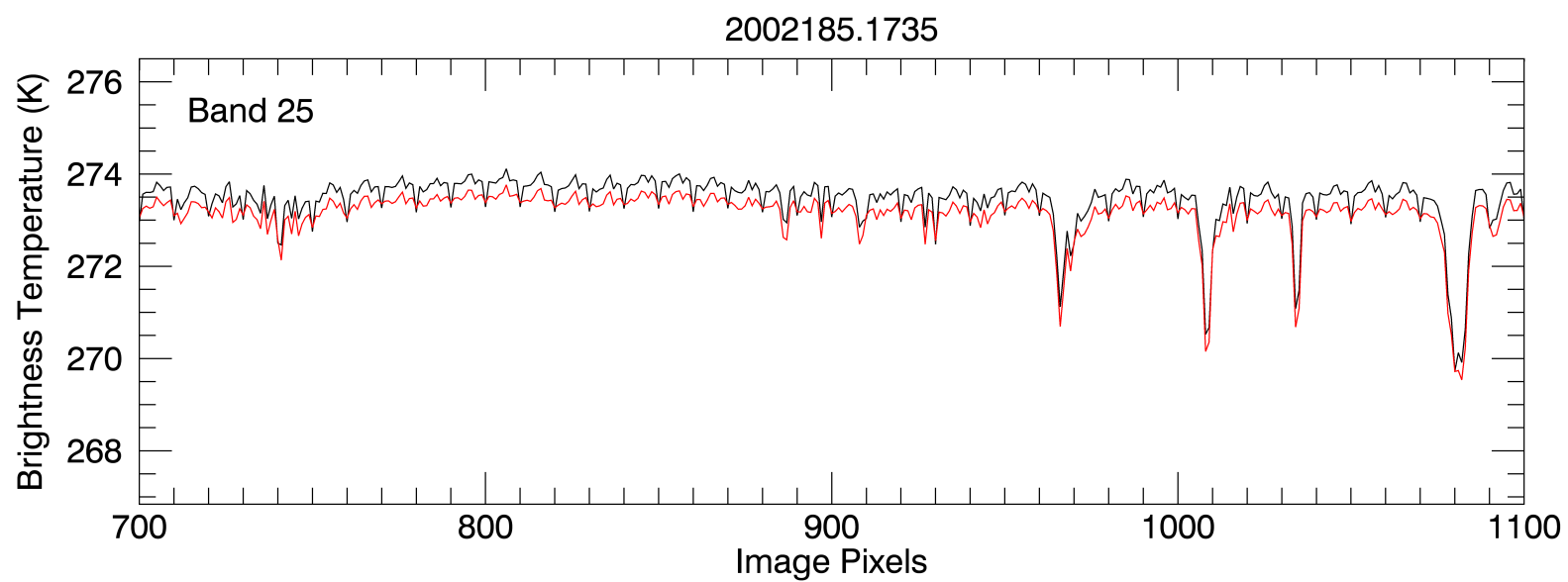

Fig. 12: Impact of the electronic crosstalk correction on the Level 1B product of receiving band 25. The image on the top left panel shows a region extracted from an L1B granule before (top) and after (bottom) the crosstalk correction is applied. The vertical lines mark the regions from where the brightness temperature profiles in the plot on the bottom panel were extracted. In the profile plots, the black line corresponds to the original L1B image and the red line, to the crosstalk corrected image. Spikes in the profiles correspond to stripes in the images. Both the images and the profiles show a reduction in the striping associated with the detector $1-$ of about $0.5 \mathrm{~K}-$ and a small overall downward radiometric shift - around $0.3 \mathrm{~K}$ - associated with the lateral ghosts. The top right panel shows detector histograms (from the entire granule, not only from the region shown in the image) before (top) and after (bottom) crosstalk correction is applied. The histogram for the detector 1 (dark red), which was previously dislocated towards lower temperatures can be seen falling into family after correction. 

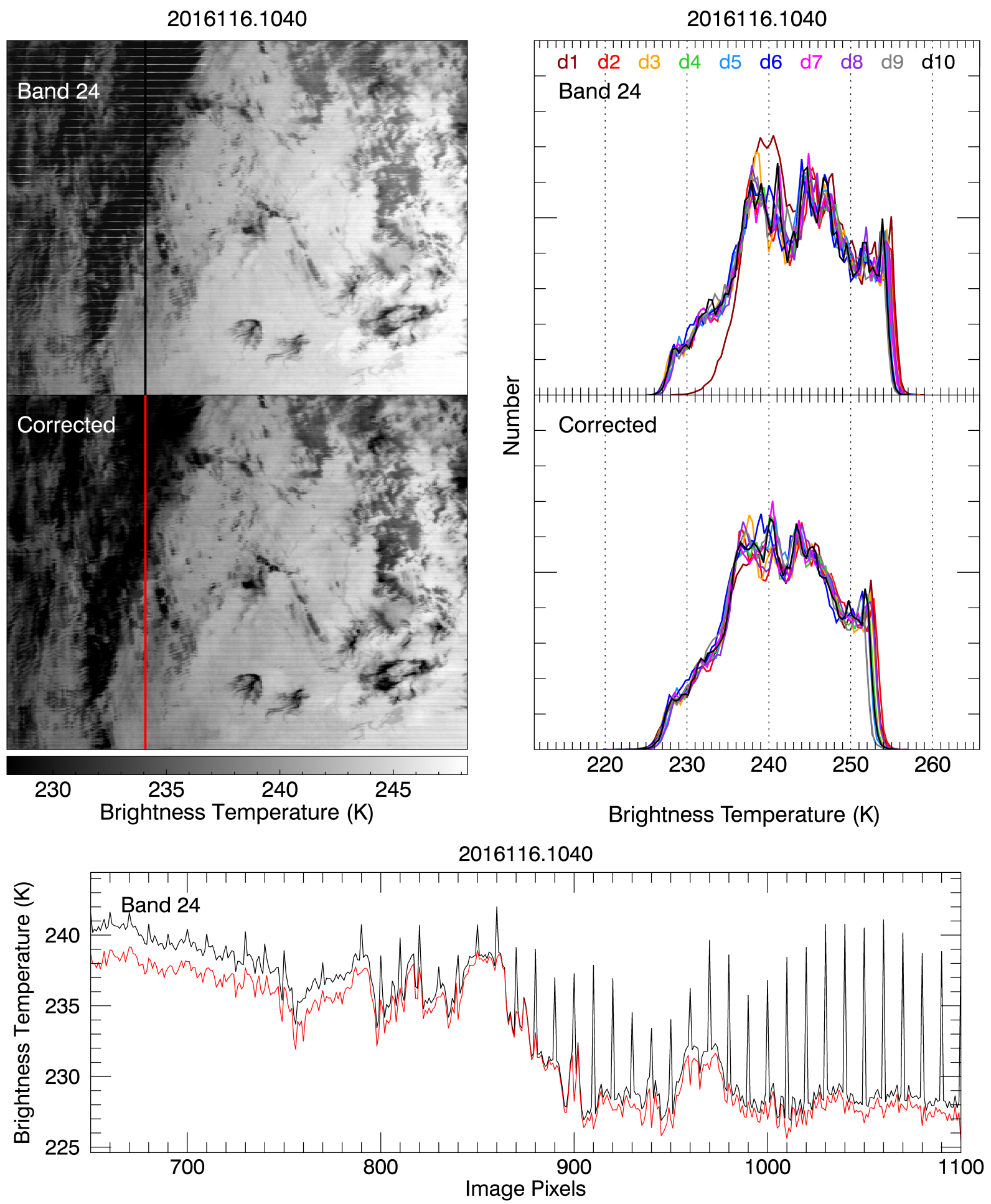

Fig. 13: Impact of the electronic crosstalk correction on the L1B product of receiving band 24. The image on the top left panel shows a region extracted from an L1B granule before (top) and after (bottom) the crosstalk correction is applied. The vertical lines mark the regions from where the temperature profiles in the plot on the bottom panel were extracted. In the profile plots, the black line corresponds to the original L1B image and the red line, to the corrected image. Spikes in the profiles correspond to stripes in the images. Both the image and the profiles show a strong reduction in the striping associated with detector 1 and a considerable overall downward radiometric shift. The top right panel shows detector histograms (extracted from the entire granule, not only the region shown in the image) before (top) and after the crosstalk correction (bottom) was applied. The histogram for the detector 1 (dark red) can be seen falling into family after correction. The histograms also show an overall downward radiometric shift after correction, of about $1 \mathrm{~K}$ at low brightness temperatures and over 2 $\mathrm{K}$ at the highest values. 
2002185.1735

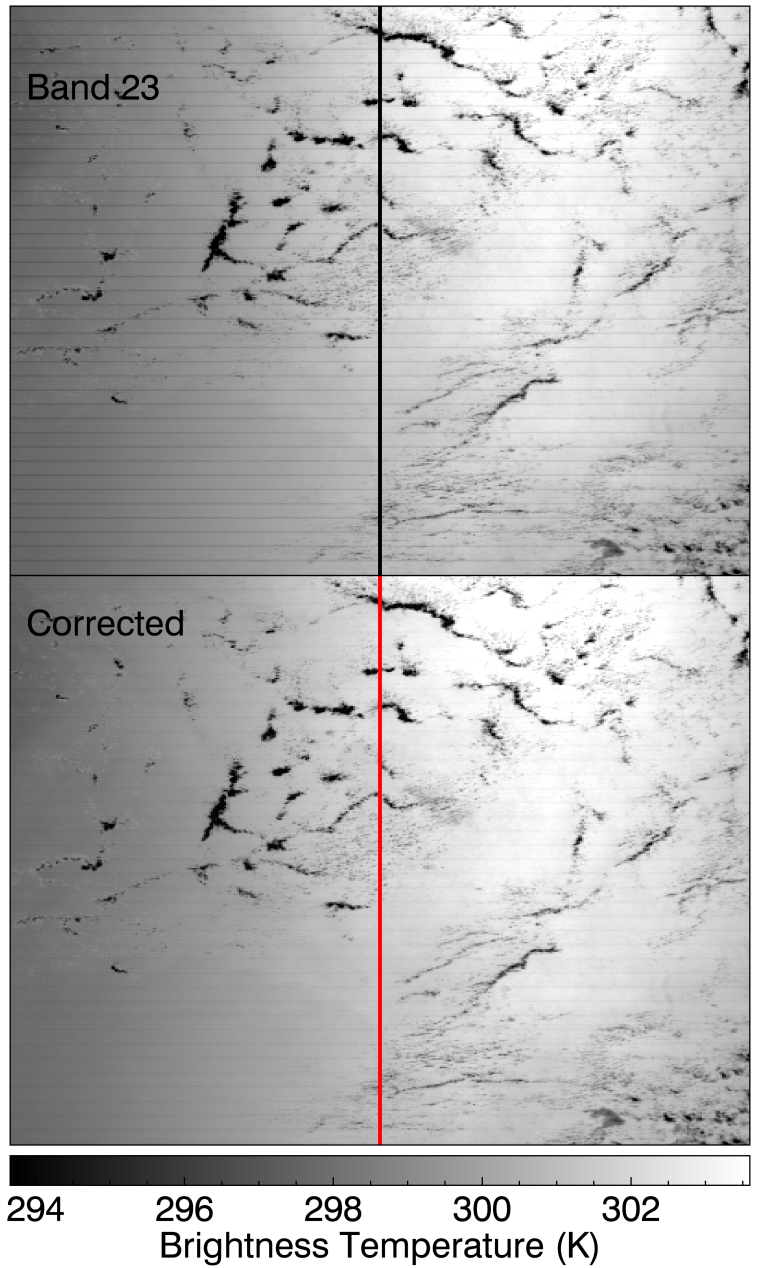

2002185.1735

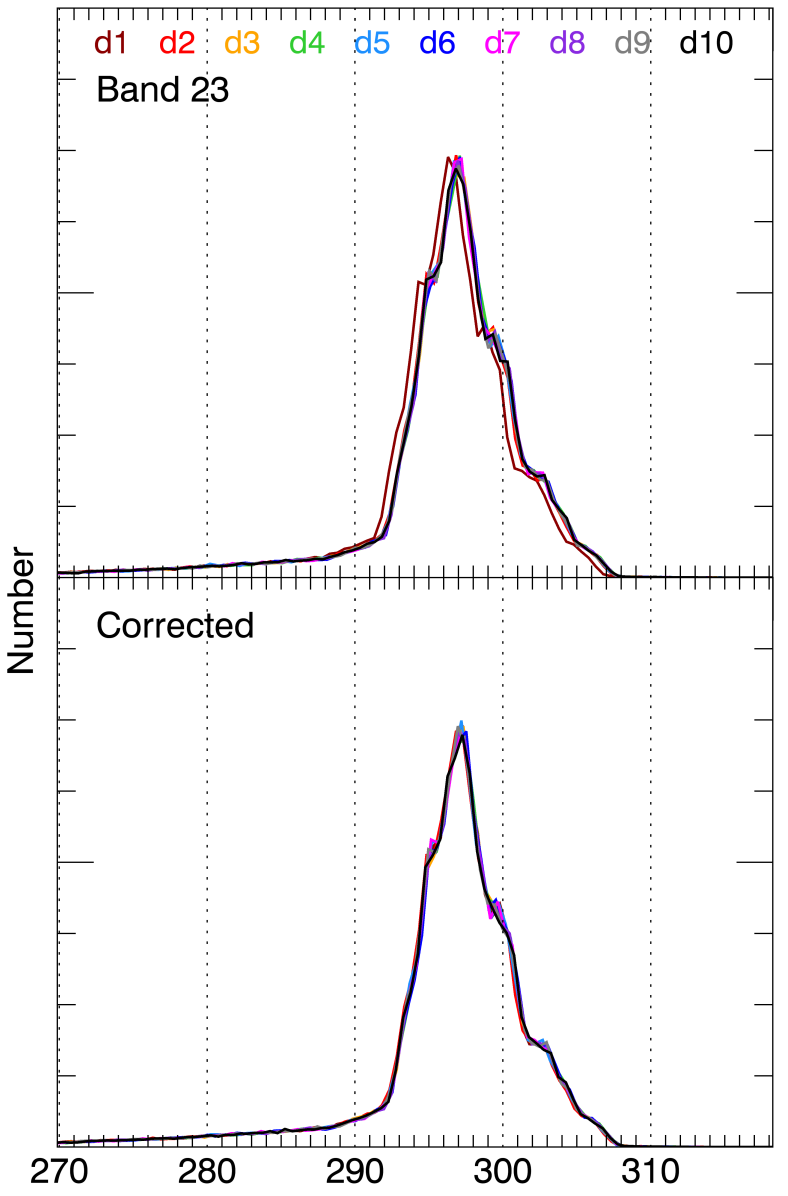

Brightness Temperature $(\mathrm{K})$

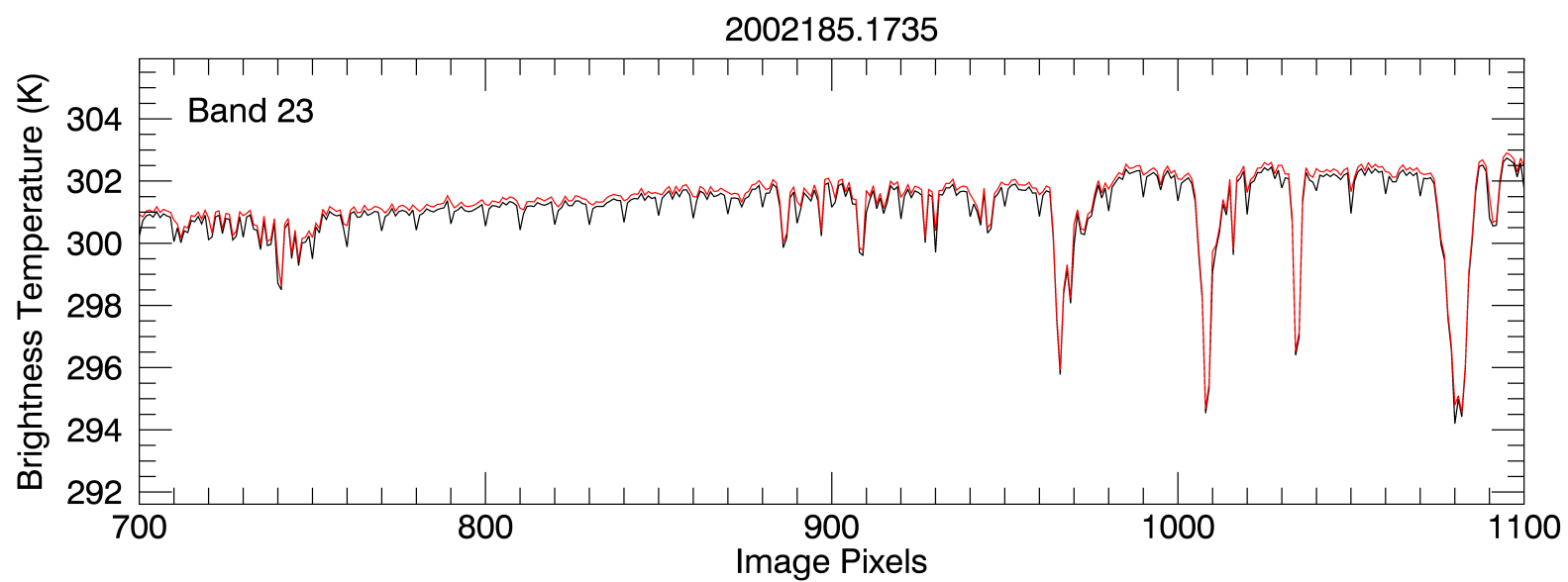

Fig. 14: Impact of the electronic crosstalk correction on the L1B product of receiving band 23. The image on the top left panel shows a region extracted from an L1B granule before (top) and after (bottom) the crosstalk correction is applied. The vertical lines mark the regions from where the temperature profiles in the plot on the bottom panel were extracted. In the profile plots, the black line corresponds to the original L1B image and the red line, to the corrected image. Both the image and the profiles show a reduction in striping associated with the detector 1 - of about $0.5 \mathrm{~K}$ - and a small overall radiometric shift - of about $0.25 \mathrm{~K}$ - associated with the lateral ghosts. The top right panel shows detector histograms (from the entire granule) before (top) and after crosstalk correction (bottom). The histogram for detector 1 (dark red) can be seen falling into family after correction. 

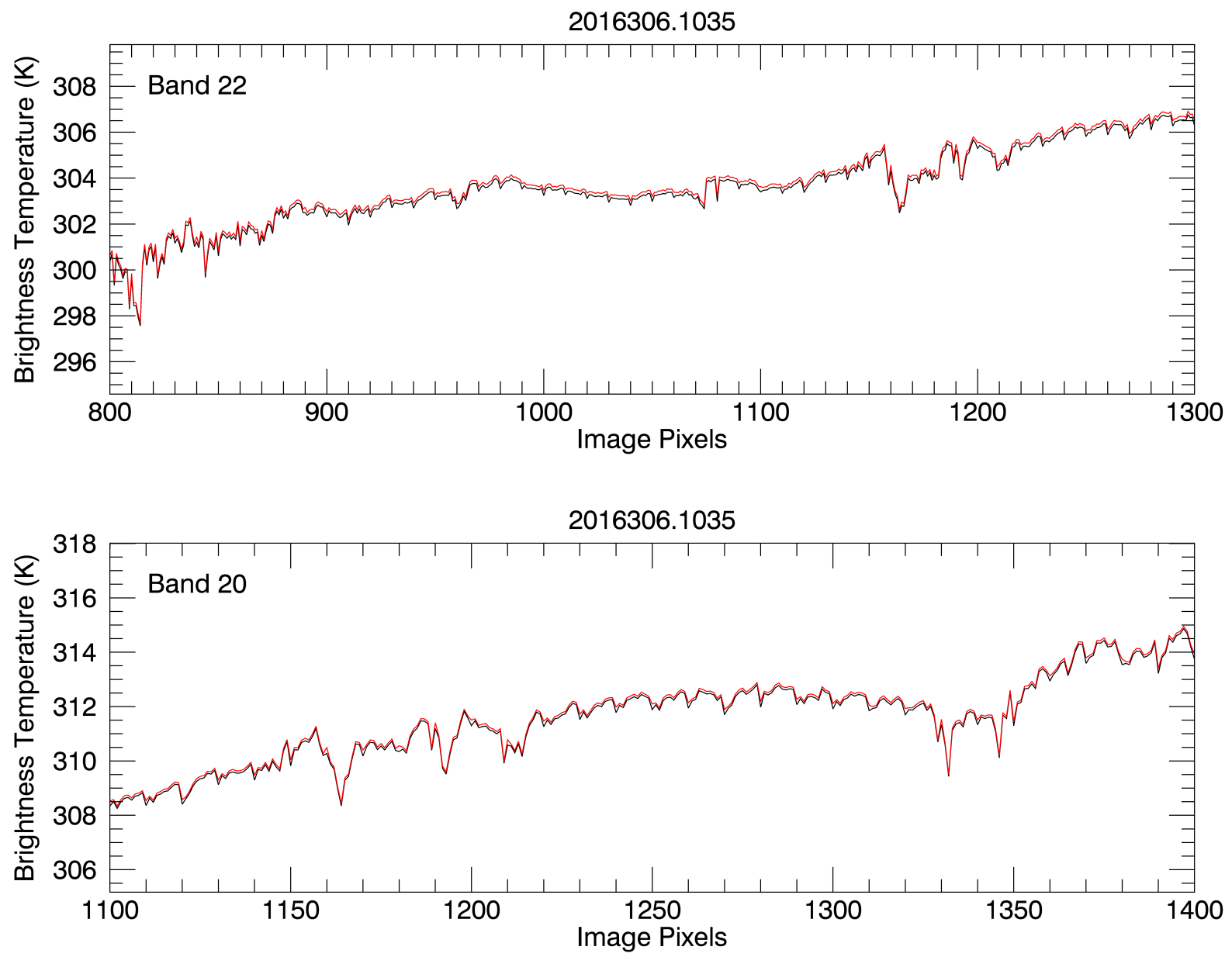

Fig. 15: Impact of the electronic crosstalk correction on the L1B products of receiving bands 22 (top) and 20 (bottom). In the profile plots, the black line corresponds to the original L1B images and the red line, to the corrected image. Both bands show a slight reduction in striping, which was already very faint and a minor radiometric shift after correction. Images and histogram plots were not shown here because they could not show such small differences.

the contamination is always the detector 10 of the respective sending band. The exception to this is an extra, fainter simple ghost that appears in lunar images by detector 1 of band 23 , which is caused by signal coming from detector 1 of the same sending band. The pattern of contamination where detector 10 of the sending band contaminates detector 1 of the receiving band is such that the signal in the receiving and sending detectors are always read out - in the electronic output - in immediate sequence. One interpretation for this pattern of contamination would be a failure in stopping the detector sampling of the receiving band in time, before the signal from the sending detector starts being read.

The lateral ghosts we have identified in lunar images by bands $20,22,23,24$, and 25 are characterized by an extended region of faint positive signal next to the main lunar image. We found they affect all the detectors of the receiving bands and are caused by signal from multiple sending detectors of multiple sending bands. We determined that the receiving bands 20,22 , and 23 are contaminated by signal from the sending bands 24 and 25 and that the receiving bands 24 and
25 receive signal from the sending bands $20,21,22$, and 23 .

We found the electronic crosstalk causing the simple and lateral ghosts to have been present since the beginning of the mission, in 2002. The crosstalk coefficients we derived from both types off ghosts are quite stable throughout the mission, with only very slight downward trends for simple ghosts in some bands.

Through the analysis of the impact of the crosstalk correction on the Level 1B product we verified the existence of two types of artifacts related to the crosstalk contamination: striping and radiometric bias. By far, band 24 is the most affected. The analyzed granules showed a downward radiometric shift reaching $2 \mathrm{~K}$ and detector 1 striping, where the response of detector 1 easily reaches values of about $10 \mathrm{~K}$ over those of the neighboring detectors. Bands 25 and 23 come next, with striping artifacts of about $0.5 \mathrm{~K}$ and radiometric shifts around 0.3 and $0.25 \mathrm{~K}$, respectively. For bands 22 and 20, both kinds of artifacts are present, but are minor.

We thus conclude by discussing which bands and detectors we consider good candidates for a possible future implemen- 
TABLE IV: Presence of artifact $(\times)$ and eligibility for possible future implementation of the correction into production $(\otimes)$.

\begin{tabular}{|c||c|c|}
\hline Band & Overall Radiometric Shift & Detector 1 Striping \\
\hline \hline B20 & $\times$ & $\times$ \\
\hline B21 & & $\times$ \\
\hline B22 & $\times$ & $\times$ \\
\hline B23 & $\times$ & $\otimes$ \\
\hline B24 & $\otimes$ & $\otimes$ \\
\hline B25 & $\times$ & $\otimes$ \\
\hline
\end{tabular}

tation of a crosstalk correction into production (summarized in Table IV). We find the detector 1 striping artifacts seen in bands 23, 24, and 25 to be good candidates, both due to the severity of the artifacts and the success of the correction. Regarding the overall radiometric shifts seen in various of the bands, we find that only in band 24 it is severe enough to compel further investigation, since, as we discussed above, overall radiometric shifts that are stable through time, are more challenging to confirm than striping, which has an obvious deleterious impact in the images. To the best of our knowledge, MODIS band 24 is not used in higher level MODIS products. Products making use of bands 23 or 25, for example, such as the short-wavelength Sea Surface Temperature product, could see some limited impact. Further investigation of the selected artifacts will be pursued by the MCST in the future.

\section{ACKNOWLEDGMENT}

The authors would like to thank other members of MCST and in particular Dr. Tiejun Chang for revising this manuscript. GRK made use of and acknowledges IDL libraries IDL Astronomy Users Library [19], pp lib [20], and mglib. Level $1 \mathrm{~A}$ data used in this work were obtained from Level-1 and Atmosphere Archive \& Distribution System (LAADS) Distributed Active Archive Center (DAAC), located in the Goddard Space Flight Center in Greenbelt, Maryland (https://ladsweb.nascom.nasa.gov/).

\section{REFERENCES}

[1] W. L. Barnes, T. S. Pagano, and V. V. Salomonson, "Prelaunch characteristics of the moderate resolution imaging spectroradiometer (MODIS) on EOS-AM1," IEEE Transactions on Geoscience and Remote Sensing, vol. 36, no. 4, pp. 1088-1100, 1998.

[2] X. Xiong, B. N. Wenny, and W. L. Barnes, "Overview of NASA earth observing systems Terra and Aqua moderate resolution imaging spectroradiometer instrument calibration algorithms and on-orbit performance," Journal of Applied Remote Sensing, vol. 3, no. 1, p. 032501, 2009.

[3] X. Xiong, B. N. Wenny, A. Wu, W. L. Barnes, and V. V. Salomonson, "Aqua MODIS thermal emissive band on-orbit calibration, characterization, and performance," IEEE Transactions on Geoscience and Remote Sensing, vol. 47, no. 3, pp. 803-814, 2009.

[4] X. Xiong, J. Sun, A. Angal, K. Chiang, and W. Barnes, "Applications and results of MODIS lunar observations," in Proc. SPIE, vol. 6744, 2007 , p. $67441 \mathrm{H}$.

[5] J. Sun and X. Xiong, "Solar and lunar observation planning for earthobserving sensor," in Proc. SPIE, vol. 8176, 2011, p. 817610.

[6] T. Wilson and X. Xiong, "Scheduling observations of celestial objects for Earth observing sensor calibration," in SPIE Remote Sensing. International Society for Optics and Photonics, 2016, pp. 1000 011-1000 011.

[7] J. Sun, X. Xiong, N. Che, and A. Angal, "Terra MODIS band 2 electronic crosstalk: cause, impact, and mitigation," in Remote Sensing. International Society for Optics and Photonics, 2010, p. 78261Y.
[8] J. Sun, X. Xiong, S. Madhavan, and B. N. Wenny, "Terra MODIS band 27 electronic crosstalk effect and its removal," IEEE Transactions on Geoscience and Remote Sensing, vol. 52, no. 3, pp. 1551-1561, 2014.

[9] J. Sun, S. Madhavan, X. Xiong, and M. Wang, "Investigation of the electronic crosstalk in Terra MODIS band 28," IEEE Transactions on Geoscience and Remote Sensing, vol. 53, no. 10, pp. 5722-5733, 2015.

[10] _ "Electronic crosstalk in Terra MODIS thermal emissive bands," in SPIE Optical Engineering + Applications. International Society for Optics and Photonics, 2015, pp. 96070V-96070V.

[11] T. Wilson, A. Wu, X. Geng, Z. Wang, and X. Xiong, "Analysis of the electronic crosstalk effect in Terra MODIS long-wave infrared photovoltaic bands using lunar images," in SPIE 10004, 2016, p. 100041C.

[12] T. Wilson, A. Wu, A. Shrestha, X. Geng, Z. Wang, C. Moeller, R. Frey, and X. Xiong, "Development and implementation of an electronic crosstalk correction for bands 27-30 in Terra MODIS collection 6," Remote Sensing, vol. 9, no. 6, p. 569, 2017.

[13] G. R. Keller, Z. Wang, A. Wu, and X. Xiong, "Aqua MODIS band 24 crosstalk striping," IEEE Geoscience and Remote Sensing Letters, vol. 14, no. 4, pp. 475-479, 2017.

[14] _ - "Aqua MODIS electronic crosstalk on SMWIR bands 20 to 26," in 2017 IEEE International Geoscience and Remote Sensing Symposium (IGARSS), July 2017, pp. 4174-4177.

[15] G. R. Keller, Z. Wang, A. Wu, and X. J. Xiong, "Aqua MODIS electronic crosstalk survey from moon observations," in Sensors, Systems, and Next-Generation Satellites XXI, vol. 10423. International Society for Optics and Photonics, 2017, p. 1042314.

[16] J.-Q. Sun, X. Xiong, W. L. Barnes, and B. Guenther, "Modis reflective solar bands on-orbit lunar calibration," IEEE Transactions on geoscience and remote sensing, vol. 45, no. 7, pp. 2383-2393, 2007.

[17] T. Wilson, A. Shrestha, and X. Xiong, "Electronic crosstalk impact assessment in the terra modis mid-wave infrared bands," in Sensors, Systems, and Next-Generation Satellites XXI, vol. 10423. International Society for Optics and Photonics, 2017, p. 104231Z.

[18] Y. Li, A. Wu, and X. Xiong, "Evaluating calibration of modis thermal emissive bands using infrared atmospheric sounding interferometer measurements," in Proc. SPIE, vol. 8724, 2013, pp. 87240X-1.

[19] W. B. Landsman, "The IDL Astronomy User's Library," in Astronomical Data Analysis Software and Systems II, ser. Astronomical Society of the Pacific Conference Series, R. J. Hanisch, R. J. V. Brissenden, and J. Barnes, Eds., vol. 52, Jan. 1993, p. 246.

[20] P. Penteado, "pp_lib v1.1," Jun. 2016. [Online]. Available: http://dx.doi.org/10.5281/zenodo.56247 